\title{
Stepwise Diagnosis and Treatment from Uninvestigated Dyspepsia to Functional Dyspepsia in Clinical Practice in Japan: Proposal of a 4-Step Algorithm
}

\author{
Shigemi Nakajima \\ Departments of Medicine, Gastroenterology and Healthcare, Social Insurance Shiga Hospital, Shiga, Japan
}

\section{Key Words}

Dyspepsia • Algorithm • Helicobacter pylori •

Gastroesophageal reflux disease $\cdot$ Functional

gastrointestinal disorder $\cdot$ Nonerosive gastroesophageal

reflux disease $\cdot$ Proton pump inhibitor - Prokinetics $\cdot \mathrm{H}_{2}$

receptor antagonist

\begin{abstract}
Background/Aim: Management of dyspepsia may differ between countries. In Japan, Helicobacter pylori infection should be considered because of the high prevalence of the infection and the related diseases. I tried to propose an algorithm for stepwise diagnosis and treatment from uninvestigated dyspepsia to functional dyspepsia in clinical practice in Japan. Methods: A proposal was made from my personal review of published studies. Results: A 4-step algorithm was proposed. In the first step, organic, systemic or metabolic diseases are excluded and nonulcer dyspepsia (NUD) is diagnosed. The second step is to test and treat $H$. pylori infection for NUD patients. In the third step, proton pump inhibitor (PPI)-responsive NUD is discriminated by PPI. Nonresponsive patients in the third step are diagnosed as having PPI-resistant NUD. In the fourth step, functional dyspepsia is diagnosed with the Rome III questionnaire. In this algorithm, it is not necessary to do special function tests for functional gastrointestinal disorders. Treatment options are automatically restricted in each step. Conclusions: The proposed 4-step
\end{abstract}

algorithm may be useful in clinical practice for the diagnosis from uninvestigated dyspepsia to functional dyspepsia in Japan. With this stepwise algorithm, treatment options are restricted in each step and the management of dyspepsia may be easier.

Copyright $\odot 2009$ S. Karger AG, Basel

\section{Introduction}

Management of dyspepsia may be different between countries because of the differences in the rate of Helicobacter pylori infection, prevalence of diseases such as peptic ulcer, gastric cancer and reflux esophagitis, medical insurance system, socioeconomic status and other factors. In the United States, for example $[1,2]$, where the rate of $H$. pylori infection is low, empirical proton pump inhibitor (PPI) therapy is recommended for patients having heartburn or regurgitation without medical tests. For patients not having such symptoms and aged 55 and younger, testing and treating $H$. pylori infection is recommended, and then PPI therapy is recommended before esophagogastroduodenoscopy (EGD). In European countries, the strategy is similar [3]. However, in countries such as Japan, where the prevalence of $H$. pylori infection is high, EGD should be recommended in the early stage of management to diagnose or exclude serious diseases. Thus, there may be differences in the management of dys-

\section{KARGER}

Fax +4161306 1234

E-Mail karger@karger.ch

www.karger.com (c) $2009 \mathrm{~S}$. Karger AG, Basel

0012-2823/09/0795-0019\$26.00/0

Accessible online at:

www.karger.com/dig
Shigemi Nakajima, MD, PhD, FISIM

Departments of Medicine, Gastroenterology and Healthcare

Social Insurance Shiga Hospital, 16-1 Fujimidai, Otsu

Shiga 520-0846 (Japan)

Tel. +81 77537 3101, Fax +81 77534 0566, E-Mail shigemin@rainbow.plala.or.jp 


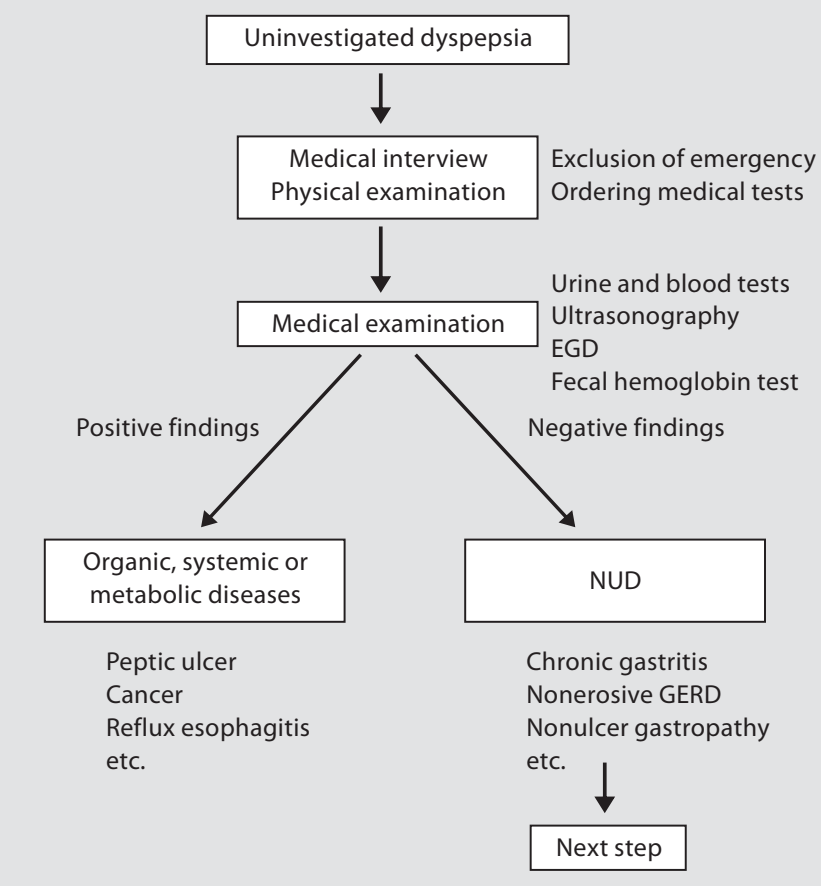

Fig. 1. The first step for dyspepsia: exclusion of organic, systemic or metabolic diseases. GERD = Gastroesophageal reflux disease.

pepsia between countries and the profiles of the diseases may be different, which means that the profiles of nonulcer dyspepsia (NUD) and functional dyspepsia (FD) may be different between countries.

FD is classified as one of the syndromes of functional gastrointestinal disorders (FGIDs), and is defined in Rome III criteria as the presence of one or more dyspepsia symptoms that are considered to originate from the gastroduodenal region in the absence of any organic, systemic or metabolic disease that is likely to explain the symptoms [4]. Although Rome III proposes a sophisticated, integrated, international classification of FGIDs, strict indication of the criteria in clinical practice is not easy because FGIDs consist of more than 20 syndromes and the diagnosis and classification for each patient is too complicated and time-consuming. In addition, the diagnosis is symptom based and needs some clinical tests to exclude organic, systemic or metabolic diseases. Therefore, most patients who are suspected of having FGIDs usually need clinical examinations or observations with certain medications. The actual indication of the criteria is not completed at the first visit of the patient with un- investigated or partially investigated dyspepsia, and stepwise diagnosis is generally performed.

In this paper, I try to organize the stepwise diagnosis and suggest a flowchart (4-step algorithm) for diagnosing and treating uninvestigated dyspepsia until FD is diagnosed in clinical practice without any special function tests for FGIDs.

Because prevalence of diseases and medical insurance systems are different among countries, this algorithm is not always indicated in every country. However, this is one of the proposed procedures in Japan where the prevalence of $H$. pylori infection and the related diseases, including peptic ulcer and gastric cancer, are high while medical expense is not high because of the medical insurance system.

A proposal is made from a personal review by the author. The author also referred to some published systematic reviews and meta-analyses. A 4-step algorithm is proposed with the author's comments as follows.

\section{The First Step: Exclusion of Organic, Systemic or}

Metabolic Diseases (fig. 1)

When an uninvestigated or partially investigated dyspepsia patient comes to an outpatient office, the first step is a medical interview followed by a physical examination. After excluding emergency cases, if gastrointestinal diseases including FGIDs are suspected, certain medical examinations are ordered to confirm diagnosis or to exclude organic, systemic or metabolic diseases. Although ordering medical tests depends on the prevalence of diseases in the country, wishes of the patient, policy of the doctor, medical insurance system or other factors, the doctor should try to make a correct diagnosis with as few tests as possible. The proposed examinations include urinalysis, blood test, ultrasonography, EGD and 2-day immunological fecal hemoglobin test. Tests for $H$. pylori infection can be ordered before EGD, a policy that is commented below. Blood test includes liver function test and pancreatic enzymes as well as routine blood cell counts and biochemical tests. For patients who have chest or epigastric symptoms, tests for heart diseases should be done. If positive hemoglobin test was obtained in 1 of the 2 fecal samples, total colonofiberscopy should be ordered. With these examinations, most organic, systemic or metabolic diseases are diagnosed. For the upper gastrointestinal tract, apparent organic diseases such as cancer, peptic ulcer or erosive reflux esophagitis must be diagnosed or excluded. Nonulcer findings in EGD such as hyperemia, erosions or mucosal hemorrhages in the stomach (nonulcer gastropathies) are not always included in the 
positive findings for organic diseases in this algorithm. Whether such gastropathies are included in organic diseases depends on the case. Those who are not diagnosed as having organic, systemic or metabolic diseases in the first step are tentatively diagnosed as NUD in this algorithm [5].

In the United States, empirical PPI treatment or testing and treating $H$. pylori infection with/without subsequent PPI treatment are recommended before EGD $[1,2]$. However, doctors who do not order EGD in the early stage of the management have to carry the risk of a delay of diagnosing serious diseases. In addition, eradication therapy before EGD has a merit only in the patients with peptic ulcer or a part of NUD patients, and such patients are few in countries where $H$. pylori infection is not prevalent. On the other hand, in countries where the prevalence of $H$. pylori infection or gastric cancer is high, EGD is recommended. H. pylori tests can be done before EGD; however, Japanese medical insurance currently does not approve $H$. pylori tests before diagnosing peptic ulcer by EGD. But if the H. pylori test is performed before EGD and negative infection is proved, PPI therapy may be indicated for young patients. However, negative results in $H$. pylori tests cannot deny EGD in countries where gastric cancer prevalence is high, because pepsinogen-positive subjects have the greatest risk for gastric cancer even if the $H$. pylori test is negative [6]. If a positive $H$. pylori test is obtained, eradication therapy may be indicated; however, gastric cancer cannot be denied without EGD. Thus, EGD is necessary, especially in older patients, whether or not a $H$. pylori test is done in advance. When EGD is performed, biopsy-based $H$. pylori tests can be done even if the patient does not have peptic ulcer but is suspected of $H$. pylori infection.

For initial treatment of uninvestigated dyspepsia, it is necessary to prescribe medicines empirically until EGD is performed. In the United Sates, empirical PPI therapy is recommended for patients having heartburn or regurgitation $[1,2]$. Although doctors in Japan can empirically prescribe PPI, EGD is recommended to avoid the delay of diagnosis as mentioned above, and many patients also want to undergo EGD because of anxiety for cancer. Because the patient may need $H$. pylori tests in the future, and the tests may show a false-negative result under treatment with PPI [7], PPI is possibly avoided until EDG is performed if the H. pylori test was not done previously. If the patient revealed to have peptic ulcer or reflux esophagitis by EGD, PPI is used as the first choice. Thus, I recommend $\mathrm{H}_{2}$ receptor antagonist as the empirical therapy in the first step in this algorithm [8]. For patients who

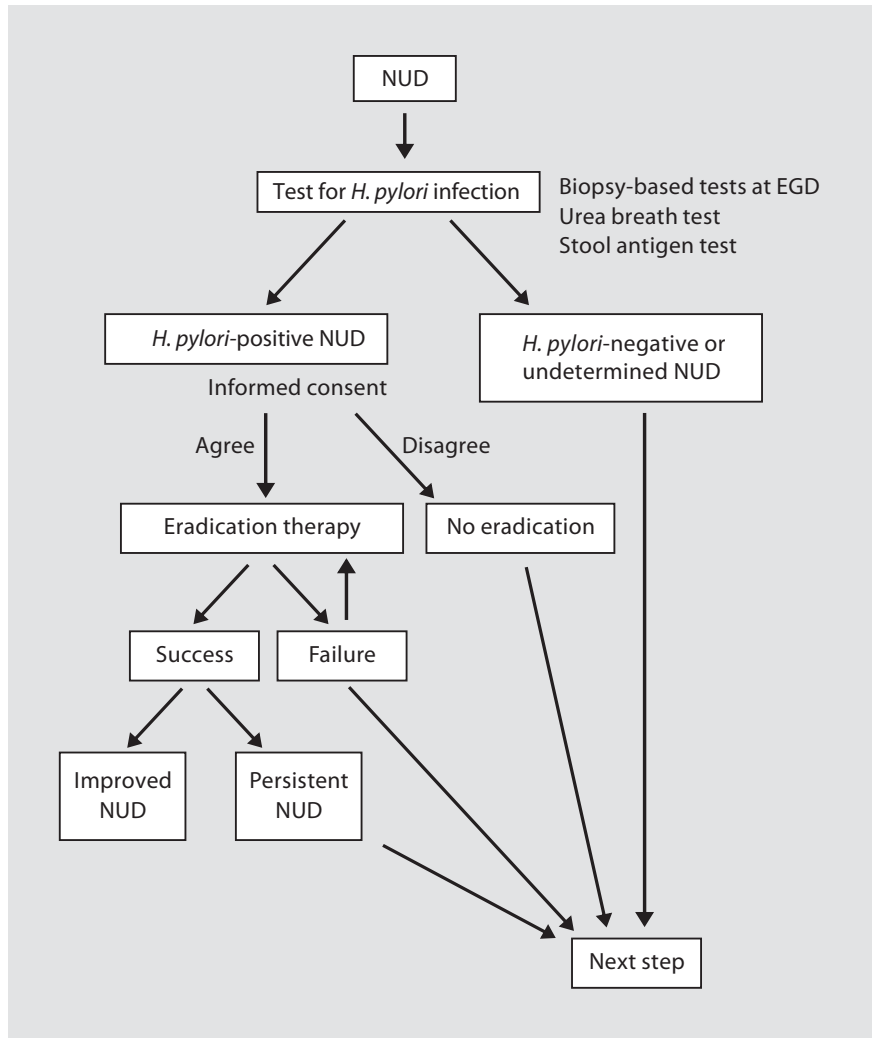

Fig. 2. The second step for dyspepsia: diagnosing $H$. pylori infection and eradication therapy.

have nonpain dyspepsia, prokinetics can be recommended [8]. Mucosal protective agents and other antiulcer drugs are also considered for use.

\section{The Second Step: Diagnosing H. pylori Infection and}

Eradication Therapy (fig. 2)

The second step is to test and treat $H$. pylori infection. Although $H$. pylori infection is not often associated with NUD or FD [9], it is a fact that eradication of the bacteria is effective in a part of dyspepsia patients [10]. If eradication is achieved, some patients do not need therapies after that, and this means that some NUD patients with $H$. $p y$ lori infection are curable with eradication therapy. This is a great benefit for the patients and for medical costs. Thus, H. pylori eradication should be considered as the first-choice therapy for $H$. pylori-infected NUD patients. In countries where the prevalence of $H$. pylori infection is high, the rate of $H$. pylori infection in NUD patients is also high [9]. Therefore, in such countries, testing and treating $H$. pylori infection in NUD patients should be considered first. If the patient is proved $H$. pylori negative 


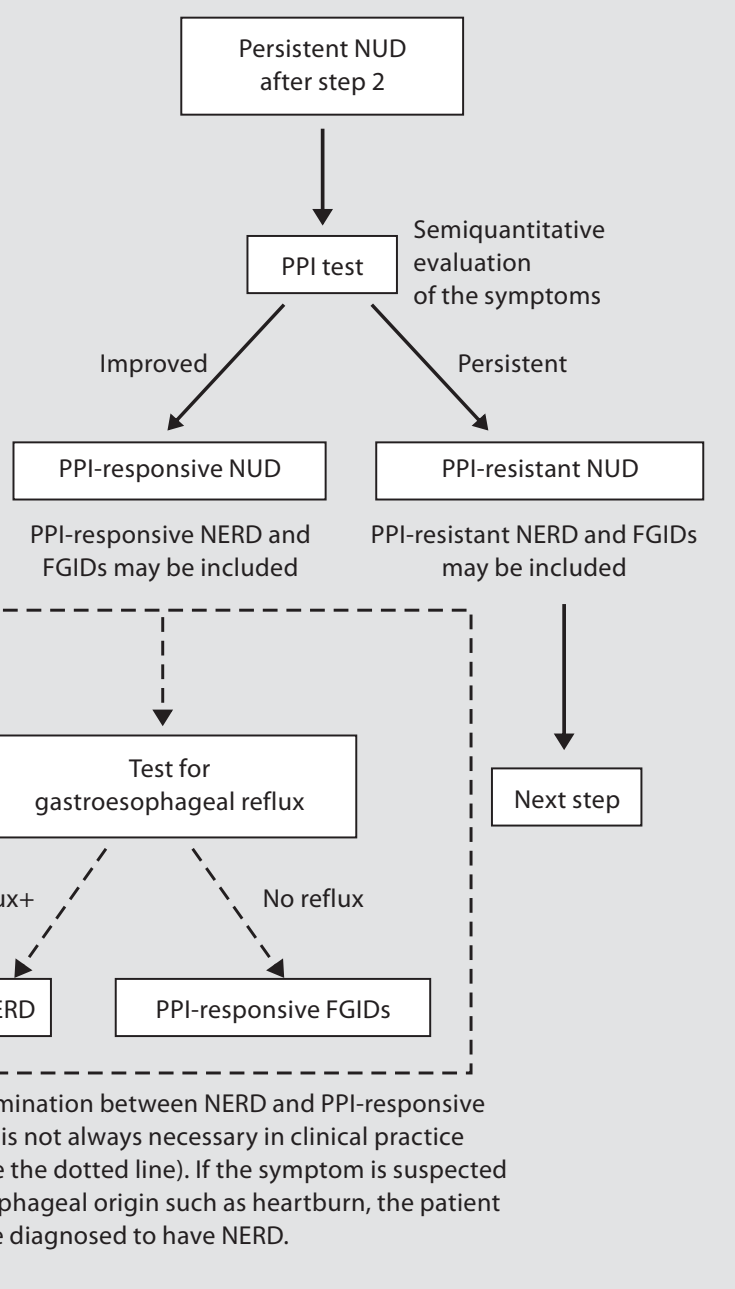

Fig. 3. The third step for dyspepsia: exclusion of PPI-responsive NUD. NERD = Nonerosive gastroesophageal reflux disease.

before this step, you can go to the third step. If not, you can go to the test for H. pylori infection.

In patients who have not been diagnosed as having $H$. pylori infection, noninvasive tests for $H$. pylori infection such as urea breath test or stool antigen test should be done. Because antibody tests with serum or urine have some false-positive results because of the previous infection, these tests can be used for screening, but are not recommended for the decision of eradication therapy. If positive $H$. pylori infection has already been diagnosed with biopsy-based tests at the previous EGD, you do not have to do H. pylori tests anymore. If this is not the case, but false-negative results of biopsy-based tests are suspected, you should consider a noninvasive $H$. pylori test.
Table 1. Information to be given to the patients who do not have peptic ulcer, but consider eradication therapy for $H$. pylori infection

\section{Information}

Frequent side effects

Not approved by medical insurance ${ }^{1}$

Eradiation rate is not $100 \%$

The disease may or may not improve after eradication therapy

Severe side effects, and what to do if they occur

Evaluation of the eradication therapy

Possible decrease in the risk of gastric cancer

Eradication may not prevent gastric cancer

Possible occurrence of reflux esophagitis

Cancer screening is still needed after H. pylori therapy ${ }^{1}$

Possible body weight gain after eradication

This consensus is not based on fully supportive evidence. This information is to help understanding for the patients to decide and avoid possible trouble after the therapy (Japanese Society for Helicobacter Research, 2007; translated and modified by the author [11]).

${ }^{1}$ Depending on countries or regions.

If the NUD patient has $H$. pylori infection, the patient should be informed of the merits and demerits of eradication therapy before prescription. For example, the Japanese Society for Helicobacter Research prepared a consensus for the information which should be given when a patient without peptic ulcer is considering eradication therapy (table 1) [11]. The consensus should also be indicated for NUD patients.

If the patient is $H$. pylori positive and has provided written consent, eradication therapy is prescribed. If the patient has been successfully eradicated and the dyspepsia symptoms have disappeared, the patient may have been cured and you do not have to go to the next step. If the eradication failed, second-line eradication therapy may be indicated. If the patient still has bothersome dyspepsia after successful eradication, you can go to the third step. You can also go to the third step if eradication failed, eradication therapy was not indicated, $H$. pylori infection was negative or diagnosis of $H$. pylori infection was not confirmed.

\section{The Third Step: Exclusion of PPI-Responsive NUD}

The third step concerns the exclusion of PPI-responsive NUD. PPI-responsive NUD includes PPI-responsive nonerosive gastroesophageal reflux disease (NERD) and 
PPI-responsive FGIDs. If the patient has heartburn or suspected esophageal symptoms and these symptoms improved with PPI therapy, the patient can be diagnosed as having NERD [12]. If the patient does not have esophageal symptoms and dyspepsia improved with PPI, the patient may have PPI-responsive FGIDs. One of the possible mechanisms of the improvement of FGIDs is that PPI decreases duodenal acid exposure which may induce dyspepsia [13]. Symptom scales are useful for objective evaluation of the therapy. For this diagnostic treatment with PPI (so-called PPI test), PPI is used at full dose for at least 2 weeks [14]. If sufficient treatment with PPI did not bring remission of symptoms, you can diagnose the patient has PPI-resistant NUD.

If you want the strict discrimination of NERD from PPI-sensitive FGIDs, special tests for gastroesophageal reflux such as the impedance test can be indicated [15]. Fortunately, a strict diagnosis is not necessary in clinical practice if the symptoms disappear. Thus, in clinical practice, if the patient improved with PPI, the patient can simply be diagnosed with PPI-responsive NUD. But in order to distinguish PPI-responsive NERD and FGIDs without function tests, the presence or absence of esophageal symptoms is the point of difference. If you furthermore want to subclassify PPI-responsive FGIDs, you have to do this with Rome III criteria. However, the Rome III integrative [16] or modular questionnaires (http://www. romecriteria.org/), have to be used before PPI therapy or after discontinuing PPI therapy.

If PPI therapy was not satisfactorily effective, the patient can be diagnosed as having PPI-resistant NUD, in which PPI-resistant NERD and PPI-resistant FGIDs are included. Then you can proceed to the next step.

\section{The Fourth Step: Diagnosis and Classification of} FGIDs (fig. 4)

If the patient has esophageal symptoms, a test for gastroesophageal reflux [15] is needed for precise diagnosis and strict discrimination of PPI-resistant NERD from FGIDs. However, most dyspepsia patients without esophageal symptoms do not require such a test. In addition, PPI-resistant NERD and FGIDs may overlap. Thus, whether or not the patient is suspected to have PPI-resistant NERD, FGIDs should be considered to diagnose PPI-resistant NUD patients.

Rome III criteria are indicated for the precise diagnosis and classification of FGIDs [4]. Because the criteria cover more than 20 syndromes, it is very difficult to diagnose and classify all FGIDs at the same time. For screening all FGIDs at the same time, integrative ques-

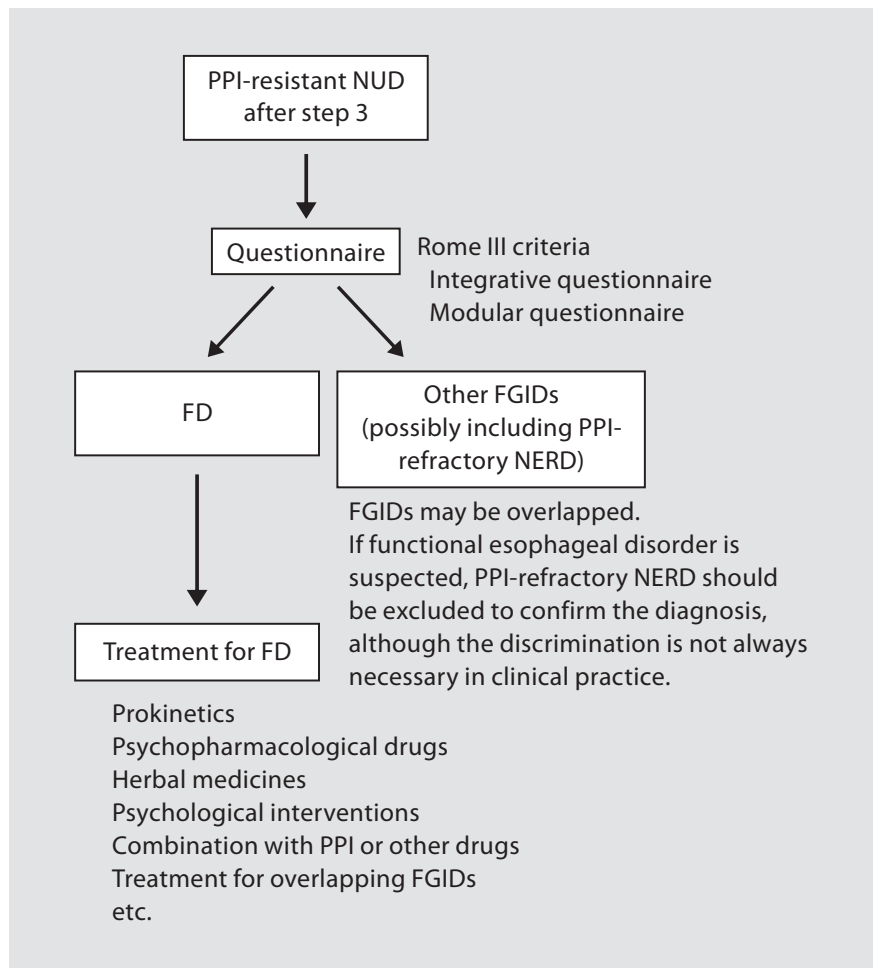

Fig. 4. The fourth step for dyspepsia: diagnosis and classification of FGIDs.

tionnaire and scoring algorithm are useful tools [16], although using the tools takes time to answer and analyze. The questionnaire consists of at least 81 questions with 19 additional questions to alarm for other serious diseases and psychosocial problems (totally 100 questions). However, if you use an automated analyzing program, analyzing answers does not take much time and, in addition, you can also diagnose overlapping FGIDs at the same time very quickly [17]. In Rome II, FD was not allowed to overlap with irritable bowel syndrome [18], but in Rome III the overlapping is allowed. In addition, FGIDs frequently overlap [17, 19]. Thus, overlapping with FD and other FGIDs is more important in Rome III than in Rome II.

For diagnosing only FD, you can use the modular questionnaire and the diagnostic criteria for FD on the Rome III website (http://www.romecriteria.org/). Because the modular questionnaire and diagnostic criteria are part of the integrated questionnaire and the scoring algorithm, the modular questionnaire can be left out if the integrative questionnaire is used. For quick screening or diagnosis of FD the modular questionnaire is useful, but it does not allow to diagnose overlapping FGIDs. If 


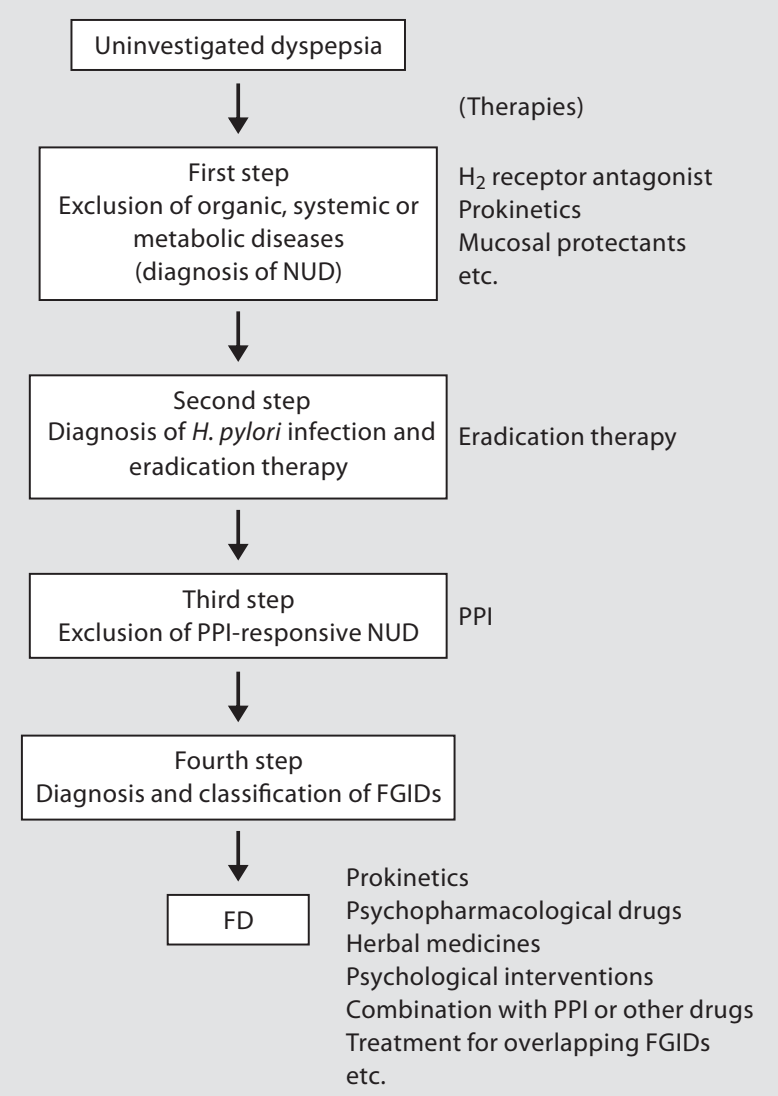

Fig. 5. Stepwise diagnosis from uninvestigated dyspepsia to FD and the step-restricted treatments.

you could not diagnose FD using only the modular questionnaire, you should consider other FGIDs, and the integrative questionnaire is recommended for use $[16,17]$.

You can also diagnose FD by using the definition in Rome III without either of the Rome III questionnaires, but you have to be careful if the patient fulfills the criteria of FD, especially for studying FD. If you do not want to study FD, you do not have to indicate the criteria too strictly in clinical practice, because the criteria in the modular questionnaire or the scoring algorithm in the integrative questionnaire are too strict.

If the patient is diagnosed as having FD, treatments are chosen. In this stepwise algorithm, you have already tried some drugs such as $\mathrm{H}_{2}$ receptor antagonist or PPI in the previous steps. So the other drugs should be considered in the fourth step. If you have not tried prokinetics in the first step, prokinetics are one of the choices in this step $[8,20]$. Because most studies on prokinetics investi- gated the efficacy of cisapride, which is now unavailable, other prokinetics such as domperidone, mosapride and itopride are considered [8]. Although some studies showed efficacy of prokinetics, it is possible that the efficacy may be drawn from publication bias [8]. Thus, it is necessary to search for other treatments if prokinetics are not effective for the patient. If the patient has psychosocial problems, psychopharmacological drugs or consultation to the specialists may be chosen for psychological interventions [21]. For depressive patients, selective serotonin reuptake inhibitors or other antidepressants may be useful. Herbal medicines such as Chinese herbal medicines or Kampo medicines (Japanese version of Chinese herbal medicines) may also be considered [22, 23]. A combination of multiple drugs may be effective. Although PPI was not effective in the third step, combination therapy with PPI may also be considered because acidification in the duodenum is one of the mechanisms of dyspepsia [13]. In my experience, $80 \%$ of FD patients diagnosed with this stepwise algorithm overlapped with the other FGIDs [17]. Thus, drugs for the other FGIDs may also be effective for the symptoms.

For patients with refractory symptoms, other organic diseases should be considered. Further examinations such as CT or PET scans may be ordered for screening malignancies. Because capsule or balloon endoscopies are available, the small intestine should be examined for the refractory patients.

\section{Summary and the Step-Restricted Treatments (fig. 5)}

My proposal of a stepwise diagnosis from uninvestigated dyspepsia to FD is summarized in figure 5 . These 4 steps look different from the algorithm recommended by the American Gastroenterological Association or the Maastricht consensus [1-3]. However, there is not much difference apart from the order of the 4 steps. Therefore, the final diagnosis of FD may be the same.

Diagnosis of FD seems difficult with the strict indication of the criteria at the first visit of the patient, but in clinical practice, it is not difficult with this stepwise diagnosis. This 4 -step algorithm does not require that you do special function tests for FGIDs. All you need are just ordinary examinations to exclude organic, systemic or metabolic diseases. Treatments of the second and third steps easily exclude curable dyspepsia with $H$. pylori eradication and controllable NUD with PPI, respectively. From the first to the third step, most diseases other than FGIDs are diagnosed or excluded. Results of the treatments from the first to the third step automatically restrict the treatment options in the fourth step. Therefore, 
in the fourth step, all you need is just the confirmation of FD and other FGIDs with adequate questionnaires, and then treatment from the restricted options. With this stepwise algorithm, treatment options are restricted in each step and the management of dyspepsia may be easier.

\section{Disclosure Statement}

The author declares that no financial or other conflict of interest exists in relation to the content of the article.

\section{References}

1 Talley NJ; American Gastroenterological Association: American Gastroenterological Association medical position statement: evaluation of dyspepsia. Gastroenterology 2005;129:1753-1755.

-2 Talley NJ, Vakil NB, Moayyedi P: American Gastroenterological Association technical review on the evaluation of dyspepsia. Gastroenterology 2005;129:1756-1780.

-3 Malfertheiner P, Megraud F, O'Morain C, Bazzoli F, El-Omar E, Graham D, Hunt R, Rokkas T, Vakil N, Kuipers EJ: Current concepts in the management of Helicobacter pylori infection: the Maastricht III consensus report. Gut 2007;56:772-781.

4 Tack J, Talley NJ, Camilleri M, Holtmann G, Hu P, Malagelada JR, Stanghellini V: Functional gastroduodenal disorders; in Drossman DA, Corazziari E, Delvaux M, Spiller RC, Talley NJ, Thompson WG, Whitehead WE (eds): ROME III: The Functional Gastrointestinal Disorders, ed 3. McLean, Degnon Associates Inc., 2006, pp 419-486.

5 Colin-Jones DG: Management of dyspepsia: report of a working party. Lancet 1988; 1 : 576-579.

6 Ohata H, Kitauchi S, Yoshimura N, Mugitani K, Iwane M, Nakamura H, Yoshikawa A, Yanaoka K, Arii K, Tamai H, Shimizu Y, Takeshita T, Mohara O, Ichinose M: Progression of chronic atrophic gastritis associated with Helicobacter pylori infection increases risk of gastric cancer. Int J Cancer 2004;109: 138-143.

7 Laine L, Estrada R, Trujillo M, Knigge K, Fennerty MB: Effect of proton-pump inhibitor therapy on diagnostic testing for Helicobacterpylori. Ann Intern Med 1998;129:547550 .

8 Moayyedi P, Soo S, Deeks J, Delaney B, Innes $M$, Forman D: Pharmacological interventions for non-ulcer dyspepsia. Cochrane Database Syst Rev 2006;2:CD001960.
9 Talley NJ, Hunt RH: What role does Helicobacter pylori play in dyspepsia and nonulcer dyspepsia? Arguments for and against $H$. $p y$ lori being associated with dyspeptic symptoms. Gastroenterology 1997;113(suppl 6): S67-S77.

10 Moayyedi P, Soo S, Deeks J, Delaney B, Harris $\mathrm{A}$, Innes $\mathrm{M}$, Oakes $\mathrm{R}$, Wilson $\mathrm{S}$, Roalfe A, Bennett C, Forman D: Eradication of Helicobacter pylori for non-ulcer dyspepsia. Cochrane Database Syst Rev 2006;2: CD002096.

11 Nakajima S, Inoue K: Consensus on the requirements in considering eradication therapy to Helicobacter pylori infection for the patients whose diseases are not approved to eradicate by medical insurance. Japn J Helicobacter Res 2007;9:75-76.

12 Fock KM, Talley NJ, Fass R, Goh KL, Katelaris $\mathrm{P}$, Hunt R, Hongo M, Ang TL, Holtmann G, Nandurkar S, Lin SR, Wong BC, Chan FK, Rani AA, Bak YT, Sollano J, Ho KY, Manatsathit S: Asia-Pacific consensus on the management of gastroesophageal reflux disease: update. J Gastroenterol Hepatol 2008;23:8-22.

13 Lee KJ, Vos R, Janssens J, Tack J: Influence of duodenal acidification on the sensorimotor function of the proximal stomach in humans. Am J Physiol Gastrointest Liver Physiol 2004;286:G278-G284.

14 Bate CM, Riley SA, Chapman RW, Durnin AT, Taylor MD: Evaluation of omeprazole as a cost-effective diagnostic test for gastro-oesophageal reflux disease. Aliment Pharmacol Ther 1999;13:59-66.

15 Wilson JA, Vela MF: New esophageal function testing (impedance, Bravo $\mathrm{pH}$ monitoring, and high-resolution manometry): clinical relevance. Curr Gastroenterol Rep 2008; 10:222-230.

16 Thompson WG, Drossman DA, Talley NJ, Walker L, Whitehead WE; Questionnaire Subcomittee: Rome III diagnostic questionnaire for the adult functional GI disorders (including alarm questions) and scoring algorithm; in Drossman DA, Corazziari E, Delvaux M, Spiller RC, Talley NJ, Thompson WG, Whitehead WE (eds): ROME III: The Functional Gastrointestinal Disorders, ed 3. McLean, Degnon Associates Inc., 2006, pp 917-951.
17 Nakajima S, Fujiyama Y: Preliminary evaluation of the Rome III diagnostic questionnaire for the Japanese adult functional gastrointestinal disorders (FGIDs) utilizing the automated analysis program made by the authors. Shoukakan Undou 2008;10:5-8.

18 Drossman DA: The functional gastrointestinal disorders and the Rome II process; in Drossman DA, Corazziari E, Talley NJ, Thompson WG, Whitehead WE (eds): ROME II: The Functional Gastrointestinal Disorders, ed 2. McLean, Degnon Associates Inc., 2000, pp 1-29.

19 Nakajima S: The spectra of functional gastrointestinal disorders (FGIDs) in a Japanese hospital outpatient department according to the ROME II Integrative Questionnaire. J Gastroenterol Hepatol 2008 (suppl), in press.

20 Hiyama T, Yoshihara M, Matsuo K, Kusunoki $\mathrm{H}$, Kamada T, Ito $\mathrm{M}$, Tanaka S, Chayama K, Haruma K: Treatment of functional dyspepsia with serotonin agonists: a metaanalysis of randomized controlled trials. J Gastroenterol Hepatol 2007;22:1566-1570.

21 Soo S, Moayyedi P, Deeks J, Delaney B, Lewis $\mathrm{M}$, Forman D: Psychological interventions for non-ulcer dyspepsia. Cochrane Database Syst Rev 2005;2:CD002301.

22 Oikawa T, Ito G, Koyama H, Hanawa T: Prokinetic effect of a Kampo medicine, Hangekoboku-to (Banxia-houpo-tang), on patients with functional dyspepsia. Phytomedicine 2005;12:730-734.

23 Tatsuta M, Iishi H: Effect of treatment with liu-jun-zi-tang (TJ-43) on gastric emptying and gastrointestinal symptoms in dyspeptic patients. Aliment Pharmacol Ther 1993;7: 459-462. 